\title{
Nanostructured Antagonist of Extrasynaptic NDMA Receptors
}

\author{
Alex Savchenko, ${ }^{\dagger \ddagger}$ Gary B. Braun, ${ }^{\S}$ and Elena Molokanoval $\|^{*}$
}

${ }^{\dagger}$ Departments of Bioengineering and Pediatrics, University of California, San Diego, California 92093, United States

${ }^{\ddagger}$ Stanford University, Stanford, California 94305, United States

${ }^{\S}$ Sanford Burnham Prebys Medical Discovery Institute, La Jolla, California 92037, United States

"Nanotools Bioscience, Encinitas, California 92024, United States

*Corresponding author: emolokanova@nanotoolsbio.com

\section{SUPPLEMENTARY INFORMATION}

\section{METHODS}

\section{Chemical synthesis and conjugation}

Gold nanoparticles were synthesized by citrate reduction of tetrachloroauric acid. Briefly, $197 \mathrm{mg}$ of $\mathrm{HAuCl}_{4}: 3 \mathrm{H}_{2} \mathrm{O}$ (Acros Organics) in $10 \mathrm{~mL}$ of Milli-Q (18.2 Ohm) water was diluted with $500 \mathrm{~mL}$ of additional water and brought to a vigorous boil. $50 \mathrm{~mL}$ of $46.5 \mathrm{mM}$ sodium citrate dihydrate (Sigma) was rapidly added and boiling was continued for 30 minutes. The resultant $\mathrm{Au}$ nanoparticle solution was cooled to room temperature. The citrate shell was displaced by a mixture of thiol-modified PEGs terminated with methoxy or carboxylate, at a nominal molar ratio of 4:1 methoxy:carboxylate. Concentrations of $\mathrm{Au}$ nanoparticles were determined by absorbance, using the plasmon peak at $520 \mathrm{~nm}$ and an extinction coefficient of $1.97 \times 10^{8} \mathrm{M}^{-1}$ $\mathrm{cm}^{-1}$. Au nanoparticles were also analyzed by transmission electron microscopy by depositing a diluted aqueous solution onto carbon-coated grids, followed by wicking and drying in a stream of nitrogen. The diameter was determined to be $13 \pm 1.4 \mathrm{~nm}$ (standard deviation, at least 500 cores) (Figure 1c).

Au nanoparticles were subsequently coated with polyethylene glycol (PEG) polymers. Thiolterminated PEG molecules create a stable brush-like layer on gold nanoparticles, with near covalent-level stability under neutral $\mathrm{pH}$ enabling long-term storage. Thiol-gold stability has led to an extensive use of PEG-thiols for in vitro and in vivo applications, particularly when long circulation time is required. To coat the particles, HS-PEG-OCH 2 kDa (Rapp Polymere, Cat\#122000-40, HS- $\mathrm{C}_{2} \mathrm{H}_{4}-\mathrm{PEG}-\mathrm{C}_{2} \mathrm{H}_{4}-\mathrm{OCH}_{3}$ ) was prepared in $95 \%$ ethanol at $24 \mathrm{mg} / \mathrm{mL}$, and HS-PEG-COOH 3 kDa (Rapp Polymere, Cat\#133000-4-32, HS- $\mathrm{C}_{2} \mathrm{H}_{4}$-CONH-PEG-O- $\mathrm{C}_{3} \mathrm{H}_{6}$ - 
$\mathrm{COOH}$ ) at $9 \mathrm{mg} / \mathrm{mL}$. $400 \mu \mathrm{L}$ of each were premixed and combined with $100 \mathrm{~mL}$ of Au citrate, depicted as reaction (i) in Figure 1. The following day the PEG-coated Au nanoparticles were purified from free ligand using Amicon centrifugal filters having a molecular weight cutoff of 100 $\mathrm{kDa}$ (washed with 0.1X PBS) and concentrated to $2 \mathrm{~mL}$ of 200 optical density (O.D.) at $520 \mathrm{~nm}$. Coupling of drug molecules was performed using 2-(N-Morpholino)ethanesulfonic acid (MES, $0.1 \mathrm{M}, \mathrm{pH} 4.3$ with $\mathrm{NaOH}$ ) as the coupling buffer. First, $100 \mu \mathrm{L} \mathrm{N}$-(3-dimethylaminopropyl)-N'ethylcarbodiimide hydrochloride (EDC, $0.16 \mathrm{M}$ in MES), was added to $300 \mu \mathrm{L}$ Au nanoparticles and incubated for 2 minutes. Then $100 \mu \mathrm{L}$ of $\mathrm{N}$-hydroxysuccinimide (NHS, in $0.26 \mathrm{M} \mathrm{MES}$ ) was added, incubated for 5 minutes, followed by $100 \mu \mathrm{L}$ of amine-containing drug (0.07 M in water). Separate conjugates were prepared from memantine $\mathrm{HCl}$, glucosamine, or amine-CF488A dye (Biotium) for fluorescence quantification of coupling efficiency. The glucosamine negative control was chosen based on similarity in size and having an amine group for coupling. After coupling proceeded for $1 \mathrm{~h}, 25 \mu \mathrm{L}$ of carbonate buffer (7.5\% solution, pH 9.3; Gibco) was added to raise the $\mathrm{pH}$. After $1 \mathrm{~h}, 50 \mu \mathrm{L}$ more of each of EDC and NHS solutions were added and reaction continued overnight. The Au conjugates were twice pelleted and washed at $21 \mathrm{k} R C F$ with 1X PBS (containing Tween 20 at a concentration of $0.005 \% \mathrm{w} / \mathrm{w}$ ), and stored at $4^{\circ} \mathrm{C}$ overnight. The following day extensive dialysis was performed into 1X PBS (without Tween 20) using a $20 \mathrm{kDa}$ Slide-a-Lyzer (Pierce), with three buffer changes over 48 hours. Quantification of coupled CF488A was determined by dithiothreitol stripping of the thiol coating ${ }^{25}$ and compared with a calibration curve of CF488A using its extinction coefficient of $70,000 \mathrm{M}^{-1} \mathrm{~cm}^{-1}$ (Biotium). The number of dyes per Au core was determined to be $45 \pm 22$ dye per Au core, $n=3$ reactions.

The AuM and Au-glucosamine nanoparticle products were diluted in DPBS (Hyclone) and filtered (0.22 $\mu \mathrm{m}$; Millipore). DLS was performed on different AuM samples using a Zetasizer Nano (Malvern Instruments). The Z-average hydrodynamic diameter was calculated by the software, and was found to be very similar for different samples ( 35 nm). To evaluate the stability of AuM, an aliquot was assessed by DLS after diluting 5-month old AuM in PBS versus $10 \%$ FBS in PBS, and a Z-average hydrodynamic diameter was calculated by the software for either condition. These results were comparable to initial as-synthesized AuM.

Further stability tests were pursued using UV-Vis spectroscopy by observing the plasmon peak shape and position that are known to be sensitive to aggregation (Figure 1e). For UV-vis measurements, we diluted AuM 10-fold in PBS and 10\% FBS in PBS, and incubated the samples overnight in a water bath at $37^{\circ} \mathrm{C}$. The AuM solutions were stable and showed activity in live-cell experiments when stored at $4^{\circ} \mathrm{C}$ in sealed polypropylene tubes (Eppendorf) for at 
least several months. Slight settling and adsorption to container walls could be reversed by brief sonication.

To further confirm the number of memantine molecules that could be attached to a $13-\mathrm{nm}$ Au nanoparticle, thiol-PEG-amine (3 kDa, Rapp Polymere) was used in place of the thiol-PEGcarboxylate in the above synthesis, with the same ratio during coating of $4: 1 \mathrm{HS}-\mathrm{PEG}-\mathrm{OCH}_{3}$ $2 \mathrm{kDa}$, to thiol-PEG-amine $3 \mathrm{kDa}$. It has been shown that thiol-PEGs of this length with $\mathrm{COOH}$ and amine termini can be co-deposited on Au nanoparticles producing reproducible percent loadings, controlled by the relative thiol ratio, and we expect little difference between amine and $\mathrm{COOH}^{25}$. The resulting Au-PEG-amine product was washed and reacted with the amine reactive dye 5/6-carboxyfluorescein succinimidyl ester (F-NHS, Thermo-Fisher). After conjugation to Au nanoparticles and purification of the final product, the surface molecules were displaced by adding an equal volume of the gold etchant $0.1 \mathrm{M} \mathrm{KCN}$ in $0.1 \mathrm{M} \mathrm{NaOH}$. After Au dissolved (and solution became colorless), the sample was centrifuged and the supernatant containing $F$ was analyzed by UV-Vis absorbance, using an extinction coefficient of $76900 \mathrm{M}^{-1} \mathrm{~cm}^{-1}$ at $494 \mathrm{~nm}$. Au nanoparticle concentration was determined on intact nanoparticles as described above (1 O.D. is $5.1 \mathrm{nM}$ nanoparticles). We estimated $50 \pm 16$ (standard deviation) F-NHS molecules were coupled per Au nanoparticle, consistent with the CF488A coupling results of $45 \pm 22$.

\section{Cell culture}

Cerebrocortical cultures were derived from embryonic day 17 Sprague-Dawley rats. Following enzymatical treatment (papain, $200 \mathrm{U} / \mathrm{ml} ; 30 \mathrm{~min}, 37^{\circ} \mathrm{C}$ ) and mechanical dissociation of cortices in minimum essential medium (Gibco), cells were plated at $50 \%$ confluence on polyL-lysine-coaled glass coverslips in Dulbecco's modified Eagle's medium with Ham's F12 and heat-inactivated iron-supplemented calf serum (HyClone) at a ratio of $8: 1:$.. Cells were grown at $37^{\circ} \mathrm{C}$ in a humidified $5 \% \mathrm{CO}_{2} / 95 \%$ air atmosphere for 2 weeks before experiments. Cell culture was routinely tested for mycoplasma contamination using a MycoAlert Kit (Lonza).

\section{Calcium imaging}

To detect changes in intracellular calcium concentration, we used Fluo-4 AM, a fluorescent calcium indicator (Life Technologies). Cultured cerebrocortical neurons (14-21 DIV) were loaded with $5 \mu \mathrm{M}$ Fluo-4AM for 45 minutes in the $\mathrm{CO}_{2}$ incubator in loading buffer (132 $\mathrm{mM} \mathrm{NaCl} ; 4.2$ $\mathrm{mM} \mathrm{KCl} ; 1.8 \mathrm{mM} \mathrm{CaCl}_{2}, 5 \mathrm{mM}$ D-glucose, $10 \mathrm{mM} \mathrm{HEPES}$; adjusted to $\mathrm{pH}$ 7.4). Subsequently, neurons were washed and left at room temperature for additional 15 minutes to achieve complete de-esterification of the dye. Imaging experiments were performed on a Zeiss Axiovert $100 \mathrm{M}$ microscope equipped with a fluorescein filter set (excitation 485/20 nm, emission 525/30 $\mathrm{nm}$ ). Images were acquired at 100-msec intervals for 2-5 minutes using a CCD camera (Cooke 
Sensicam). During experiments, neurons were either left untreated (to monitor spontaneous activity) or stimulated with $200 \mu \mathrm{M}$ NMDA and $10 \mu \mathrm{M}$ glycine (to trigger NMDAR-mediated calcium influx). We concluded that spontaneous calcium oscillations are synaptically driven, because $1 \mu \mathrm{M}$ tetrodotoxin (TTX) completely blocked them, while $50 \mu \mathrm{M}$ bicuculline increased their frequency. All compounds were directly applied to the recording chamber. Acquisition and analyses were performed using SlideBook 5.0 software (Intelligent Imaging Innovations). Fluorescence intensity was measured over the entire cell body, and responses were presented as arbitrary units $(\mathrm{AU})$.

\section{Electrophysiology}

Whole-cell voltage-clamp recordings were performed from primary cerebrocortical neurons after 2 weeks in vitro. The extracellular solution was comprised of (in $\mathrm{mM}$ ) $\mathrm{NaCl}, 135 ; \mathrm{KCl}, 2.5$; $\mathrm{CaCl}_{2}$, 2; $\mathrm{NaHCO}_{3}, 1 ; \mathrm{Na}_{2} \mathrm{HPO}_{4}, 0.34 ; \mathrm{KH}_{2} \mathrm{PO}_{4}, 0.44 ;$ glucose, 20; HEPES, 10; and glycine, 0.01 $(\mathrm{pH}$ 7.4). Patch electrodes with a final tip resistance of 4-7 $\mathrm{M} \Omega$, were filled with a solution containing (in $\mathrm{mM}$ ) $\mathrm{CsCl}, 120$; tetraethylammonium chloride, 20; HEPES, 10; EGTA, 2.25; $\mathrm{CaCl}_{2}, 1 ; \mathrm{MgCl}_{2}, 2$ (pH 7.4). All recordings were acquired using Digidata 1322 interface, Axopatch 200B amplifier, and pClamp software (Molecular Devices). Experiments were performed at room temperature $\left(18-22^{\circ} \mathrm{C}\right)$ at a holding potential of $-70 \mathrm{mV}$. Currents were digitally sampled at $10 \mathrm{kHz}$ and filtered at $2 \mathrm{kHz}$.

In some experiments, we monitored spontaneous activity of neuronal networks in the presence and absence memantine and AuM. In these experiments, spontaneous currents were synaptically driven, because a) these currents were completely blocked by $1 \mu \mathrm{M}$ tetrodotoxin (TTX), b) $50 \mu \mathrm{M}$ bicuculline increased their frequency, and c) co-application of $100 \mu \mathrm{M} 4$ aminopyridine (4-AP) and $50 \mu \mathrm{M}$ bicuculline increased their frequency even further. We found no correlation between the amplitude of SEPSCs and their kinetics in our experiments, indicating reliable voltage clamp.

Solutions were delivered into a $100-\mu$ l experimental chamber using a fast valve-controlled perfusion system (ALA Scientific Instruments, Inc.) at $1 \mathrm{~mL} / \mathrm{min}$, and removed by continuous suction. The performance of our perfusion system was validated on numerous occasions using various drugs at different concentrations. The application speed limit of our system as estimated by analyzing the on/off kinetics of NMDAR-mediated currents (see Fig. 4) is $\sim 50 \mathrm{msec}$. For AuM, we were able to directly monitor its successful delivery to the experimental chamber in every experiment by taking advantage of the fact that AuM solutions are exhibiting a rich red color displayed in a strong concentration-dependent manner. 
Prior to the analysis of AuM effects on NMDAR-mediated components of sEPSCs, we employed the temporal separation approach that takes advantage of different temporal activation profiles of ion channels and receptors involved in complex responses. Previously, this method was successfully used for quantification of memantine effects ${ }^{9}$. Compared to pharmacological separation, temporal separation strategy is especially advantageous in studies involving new chemical entities, because it allows avoiding potential interference with other pharmacologically active compounds. Since NMDARs and AMPA receptors (AMPARs) have drastically different kinetic parameters ${ }^{27,28}$, "temporal" separation of AMPAR- and NMDARmediated components allowed us to very reliably isolate a "slow" (i.e., NMDAR-mediated) component of a sEPSC for further analysis. First, we excluded initial transient components of sEPSCs, as they represent "fast" AMPAR-mediated currents. Then, we determined the frequency and amplitude of NMDAR-mediated sEPSCs for each experimental condition during 60-90 seconds. The mean amplitude was calculated by averaging the amplitude of the maximal NMDAR-mediated current in each burst. Subsequently, the values for charge transfer of each NMDAR-mediated SEPSC were calculated as the area under the curve starting from the peak of the first NMDAR-mediated SEPSC and continuing to the baseline. The area under the curve for occasionally occurring superimposed spikes was not included in the final values of charge transfer calculations. The sum of the charge transferred during individual bursts for each condition was normalized to the control value for that same cell. To examine the spontaneous synaptic activity at the equilibrium, the initial $5 \mathrm{sec}$ of each drug application was excluded from analysis. Normalization of all parameters was performed for each neuron to minimize intercellular experimental variations.

To evaluate whether memantine molecules conjugated to $A u$ nanostructures can get trapped inside NMDARs after closing NMDARs following the NMDA washout, we executed the following perfusion sequence: control solution, $200 \mu \mathrm{M}$ NMDA (*), $200 \mu \mathrm{M}$ NMDA plus AuM, washout with control solution for at least $30 \mathrm{sec}, 200 \mu \mathrm{M}$ NMDA $\left(^{*}\right)$, control solution. Then we compared the amplitudes of two NMDAR-mediated currents marked with asterisks in the perfusion sequence. If these amplitudes are similar, then no trapping has occurred.

Dose-response curves for AuM inhibition were determined by measuring steady-state NMDAR-mediated currents in the presence of AuM at various concentrations and normalizing them to NMDAR-mediated currents in the absence of AuM. Dose-response data were fitted by Origin software using the Marquardt-Levenberg iteration algorithm with a standard Hill equation.

To evaluate effects of AuM on eNMDARs alone, we pharmacologically isolated them from the global population of NMDARs by selectively blocking sNMDARs using the established 
protocol ${ }^{7,9}$. First, we treated neurons (5-min application) with $50 \mu \mathrm{M}$ bicuculline that by blocking GABA receptors decreased inhibitory GABA-mediated input and indirectly increased activation of sNMDARs. Then, we blocked sNMDARs by 15 -min application of $10 \mu \mathrm{M} \mathrm{MK}-801$, a longlasting NMDAR inhibitor. Since eNMDARs are closed during this treatment, they remain unaffected by MK-801. After washout of all inhibitors, sNMDARs are still blocked, while eNMDARs are available for further experiments. Our experiments with eNMDARs never exceeded $45 \mathrm{~min}$ in order to avoid eventual unbinding of MK-801 from sNMDARs ${ }^{35}$ and subsequent indiscriminative binding of MK-801 to both sNMDARs and eNMDARs.

To construct current-voltage $(\mathrm{I}-\mathrm{V})$ curves, neurons were initially clamped at $-70 \mathrm{mV}$, and then 200-ms voltage steps in $20-\mathrm{mV}$ increments were applied from $-90 \mathrm{mV}$ to $+40 \mathrm{mV}$ after NMDARmediated currents reached their steady-state amplitude. I-V curves in the absence of NMDA were subtracted from I-V curves acquired during the steady-state currents elicited by $200 \mu \mathrm{M}$ NMDA or $200 \mu \mathrm{M}$ NMDA plus AuM. The time courses of NMDAR-mediated currents during application and washout of AuM at various concentrations (namely, blocking and unblocking processes) were fitted with a single exponential function, yielding the binding and unbinding time constants ( $\tau_{\text {on }}$ and $\tau_{\text {off }}$, respectively).

\section{Cell Viability Assays}

Cell viability was assessed using the LIVE/DEAD® Viability/Cytotoxicity Kit (Life Technologies) that contains membrane-permeable calcein-AM and membrane-impermeable Ethidium homodimer-1 (EthD-1). After dye loading, live cells with intact cell membrane and high levels of enzymatic activity appear green due to enzymatic conversion of non-fluorescent calcein-AM into bright green calcein and rejection of EthD-1. In contrast, EthD-1 easily enters dead cells with compromised membrane and produces bright red fluorescent signal upon binding to nucleic acids.

In our experiments, cerebrocortical neuronal cultures were divided into four groups and treated for 1 hour with the control solution, $200 \mu \mathrm{M}$ NMDA alone, and $200 \mu \mathrm{M}$ NMDA in combination with either $10 \mu \mathrm{M}$ memantine or $50 \mathrm{nM}$ AuM. All solutions included $10 \mu \mathrm{M}$ glycine and $1 \mathrm{mM} \mathrm{MgCl}_{2}$. Each treatment group contained five blindly allocated coverslips. Twenty-four hours after the treatment, neurons were loaded for $30 \mathrm{~min}$ at $37^{\circ} \mathrm{C}$ with $2 \mu \mathrm{M}$ EthD-1, $1 \mu \mathrm{M}$ calcein $\mathrm{AM}$, and $2 \mu \mathrm{M}$ Hoechst 33342. After washout of dyes, images were randomly taken from each coverslip using the fluorescent microscope in a multichannel acquisition mode with a standard fluorescein optical filter (for calcein), a rhodamine optical filter (for EthD-1), and a DAPI optical filter (for Hoechst). The total number of cells assessed for each treatment exceeded 
1000. The cell death was calculated as the percentage ratio of the number of EthD-1-positive cells divided by the sum of the numbers of EthD-1-positive cells and calcein-positive cells.

\section{Oxygen-Glucose Deprivation}

Oxygen-glucose deprivation (OGD) is routinely used as an ischemic stroke model. Acute hippocampal slices were prepared from 4-week-old Swiss Webster mice $(n=6)$ (Hilltop Lab Animals). Mice were deeply anesthetized with isoflurane and decapitated, and the brain was rapidly removed. Slices were cut with a vibratome VT-1200 (Leica Microsystems) in chilled cutting solution, containing (in $\mathrm{mM}$ ): $\mathrm{KCl}, 3 ; \mathrm{NaH}_{2} \mathrm{PO}_{4}, 1.25 ; \mathrm{CaCl}_{2}, 0.2 ; \mathrm{MgSO}_{4}, 6 ; \mathrm{NaHCO}_{3}, 26$; sucrose, 220; glucose, 10 ( $\mathrm{pH} \mathrm{7.35).} \mathrm{Then} \mathrm{slices} \mathrm{were} \mathrm{transferred} \mathrm{into} \mathrm{oxygenated} \mathrm{artificial}$ cerebrospinal fluid (ACSF) containing (in $\mathrm{mM}$ ): $\mathrm{NaCl} 126, \mathrm{KCl}, 3 ; \mathrm{NaH}_{2} \mathrm{PO}_{4}, 1.25 ; \mathrm{CaCl}_{2}, 2$; $\mathrm{MgSO}_{4}, 1 ; \mathrm{NaHCO}_{3}, 26$; and glucose, $10(\mathrm{pH} 7.35)$, incubated at $34{ }^{\circ} \mathrm{C}$ for at least 1 hour before experiments, and were randomly placed in 24-well plates (one slice per well).

Prior to OGD studies, three groups of slices were pre-treated pharmacologically. To evaluate the contribution of eNMDARs in OGD-triggered neurotoxicity, one set of slices was pre-treated for $15 \mathrm{~min}$ in oxygenated glucose-containing ACSF containing the mixture of $10 \mu \mathrm{M}$ MK-801 and $50 \mu \mathrm{M}$ bicuculline. Two other sets of slices were pre-treated for 15 min with either $10 \mu \mathrm{M}$ memantine, or $50 \mathrm{nM}$ AuM. After pre-treatment, all slices were thoroughly washed in inhibitor-free ACSF before proceeding to the next stage of experiments. To implement an OGDmodel of ischemic stroke, slices were transferred into ACSF that contained sucrose instead of glucose at the equimolar concentration, and was equilibrated with $95 \% \quad \mathrm{~N}_{2} / 5 \% \quad \mathrm{CO}_{2}$. The treatment medium for two sets of slices included $10 \mu \mathrm{M}$ memantine, or $50 \mathrm{nM}$ AuM. During OGD treatment, slices were placed for $45 \mathrm{~min}$ in an incubator in a sealed chamber filled with $95 \% \mathrm{~N}_{2} / 5 \% \mathrm{CO}_{2}$. OGD was terminated by replacing the treatment medium with oxygenated glucose-containing ACSF. Then slices ( $n=7$ per each group) were left to recover for 6 hours before the further testing. At the same time, control slices $(n=6)$ were continuously maintained in oxygenated ACSF at $32^{\circ} \mathrm{C}$. Additional set of control slices $(n=3)$ was treated with $50 \mathrm{nM}$ AuM for 1 hour.

Cell death was quantified by measuring the release of lactate dehydrogenase (LDH) into ACSF. After the recovery period, samples of supernatant were collected from each well and tested using a colorimetric assay (Pierce LDH Cytotoxicity Assay Kit, ThermoFisher Scientific) according to the manufacturer's protocol. The levels of LDH released into ACSF were measured using a microplate reader (Infinite M200 PRO, Tecan) and expressed as a percentage of the total LDH activity in untreated OGD slices.

\section{Brain penetration study}


Brain tissue penetration distance of AuM solution was estimated by immersion of slices into concentrated AuM solution and processing for histological analysis, aided by autometallographic staining of the gold. Balb/C female mice (4-6 weeks old, Harlan Laboratories) were perfused with Dubelco PBS (Gibco) through the heart while under deep anesthesia (Avertin). The brain of each mouse was removed, washed with PBS, and cut into $\sim 2 \mathrm{~mm}$ thick coronal slices using a plastic slice-support matrix. The tissue was sliced at a thickness of $2 \mathrm{~mm}$ to allow sufficient depth for AuM brain penetration, to be able to expose both sides to the AuM solution. Slices were immediately immersed into $0.5 \mathrm{~mL} 400 \mathrm{nM}$ AuM in oxygenated ACSF (bubbled with $95 \% \mathrm{O}_{2} / 5 \% \mathrm{CO}_{2}$ ) and incubated for $30 \mathrm{~min}$. Three washes in rapid succession with buffer removed the excess AuM, and tissues were immediately frozen on dry ice in TissueTek O.C.T. compound. Then $7 \mu \mathrm{m}$ thick sections were cut and transferred to glass slides. Cryosectioning was performed in a plane perpendicular to the tissue surface to observe the decrease of AuM penetration with depth. To aid visualization of AuM in brightfield imaging (Leica DMIRE2) the sections were exposed to LI Silver Enhancement kit (Life Technologies) for 40-45 min, which deposits silver onto the Au. The reaction was quenched with $0.1 \mathrm{M}$ sodium thiosulfate, washed briefly in water, dehydrated through ethanol and xylene, and mounted in DPX Mountant (Sigma). When compared with the untreated control slices, the AuM-treated slices appeared darkly-stained due to the presence of silver-enhanced Au NPs.

Linear profiles of brightness intensity were measured (ImageJ software) from the edge of the cut tissue inwards, and normalized to the intensity furthest inside the tissue for each profile. To create each linear profile, 75 neighboring parallel line profiles were averaged together to create a mean profile. Six profiles were collected from two mice. Brightest intensity is assumed to be of a negligible AuM concentration. Normalized profiles were fit to the function Brightness = $A-B^{*}\left(\operatorname{erfc}\left(x /\left(2^{*}\left(D^{*} t\right)^{0.5}\right)\right)\right.$, where $A$ is the maximum brightness intensity (normalized and constrained to value of 100 ); $B$ is the reduced intensity at the tissue edge (dark due to the metal deposits, typical $B$ value of $\sim 40$ ); erfc is the complementary error function relevant to a Fickdiffusion scenario of constant bath concentration outside the tissue; $x$, the distance in microns from the tissue edge; $D$, the diffusion constant; and $t$, time in seconds. This formula was modified from equation 12 for one-dimensional diffusion. Note that under hypoxic conditions the dimensions of the extracellular space in the brain are decreased ${ }^{32}$, and therefore the diffusion constant could be underestimated in the experimental settings.

\section{Dendritic Spine Analysis}

Thy1-YFP-H transgenic mice (10 days old) (The Jackson Laboratory) were used ( $\mathrm{n}=5$ per experiment) to prepare organotypic hippocampal slices using the interface method. Brains were 
dissected under isoflurane anesthesia, and 350- $\mu \mathrm{m}$-thick hippocampal slices were collected in ice-cold dissection buffer containing $212 \mathrm{mM}$ sucrose, $3 \mathrm{mM} \mathrm{KCl}, 5 \mathrm{mM} \mathrm{MgCl}, 0.5 \mathrm{mM} \mathrm{CaCl}_{2}, 1$ $\mathrm{mM} \mathrm{NaH}_{2} \mathrm{PO}_{4}, 26 \mathrm{mM} \mathrm{NaHCO}_{3}$, and $10 \mathrm{mM}$ D-glucose. Dissection buffer was bubbled with $95 \%$ $\mathrm{O}_{2} / 5 \% \mathrm{CO}_{2}$. Slices were transferred onto a Millicell cell-culture insert (Millipore) and placed in medium containing Eagle's Minimum Essential Medium (50\% (vol/vol)), horse serum (25\% (vol/vol)), Earle's Balanced Salt Solution (18\% (vol/vol)), Amphotericin B $(2.5 \mu \mathrm{g} / \mathrm{mL})$, streptomycin sulfate $(100 \mu \mathrm{g} / \mathrm{mL}), 35 \mathrm{mM}$ D-glucose, $25 \mathrm{mM}$ HEPES, and $2 \mathrm{mM}$ glutamine. After 24 hours, and every 2-3 days thereafter, the culture medium was changed to medium of the similar composition but containing only $5 \%$ horse serum (vol/vol). Human synthetic $A \beta_{1-42}$ (Anaspec) was dissolved following established procedures. For 10 days, slices were treated with solution containing $250 \mathrm{nM}$ oligomeric $A \beta_{1-42}$, alone or with $10 \mu \mathrm{M}$ memantine, or $50 \mathrm{nM}$ AuM, replenished with each medium replacement. Subsequently, slices were fixed in $4 \%$ paraformaldehyde. To ensure unbiased evaluation of the dendritic spine density, all coverslips were coded to perform subsequent quantitative analysis in a blind fashion. Image stacks of YFP-expressing neurons were acquired using a 63X oil-immersion objective on a Zeiss Axiovert $100 \mathrm{M}$ microscope. In YFP-expressing neurons, three distinct fields of secondary or tertiary dendrites at least $20 \mu \mathrm{m}$ in length were selected randomly and analyzed using SlideBook 5.0 software. Spines were identified on dendritic branches of the same and counted through $z-$ stacks along dendrites in the 3-D space without knowledge of treatment. The spine density was presented as the number of dendritic spines per $1 \mu \mathrm{m}$ of the dendrite length. All animal care and experimental procedures were performed in compliance with ethical regulations and in accordance with guidelines and regulations approved by the Institutional Animal Care and Use Committee (IACUC) of the Sanford Burnham Prebys Medical Discovery Institute and the University of California.

\section{Statistical analysis}

Data were expressed as mean \pm SEM. We performed the Shapiro-Wilk test on our data sets to test the normality of the data distribution. The dataset is considered normally distributed if the test cannot reject normality at the 0.05 level. Results were interpreted using one-way ANOVA with a Bonferroni post-hoc test, or unpaired a Student's $t$-test, when appropriate. The variance was similar between the groups that were being compared. Differences were considered statistically significant at $P<0.05$. We determined the sample sizes in our experiments for the statistical power of 0.8 , the statistical significance criteria of 0.05 , and the pre-specified effect size of 0.2 by performing "Power and Sample Size Analysis" calculations using OriginPro 2015 software. 